\title{
Predictors of Adverse TB Treatment Outcome among TB/HIV Patients Compared with Non-HIV Patients in the Greater Accra Regional Hospital from 2008 to 2016
}

\author{
Kenneth Mawuta Hayibor $\mathbb{D}^{1,2}$ Delia Akosua Bandoh $\mathbb{D}^{1,2}$ Adwoa Asante-Poku $\left(\mathbb{D},{ }^{1}\right.$ \\ and Ernest Kenu ${ }^{2}{ }^{2}$ \\ ${ }^{1}$ Noguchi Memorial Institute for Medical Research, University of Ghana, Ghana \\ ${ }^{2}$ School of Public Health, University of Ghana, Ghana \\ Correspondence should be addressed to Ernest Kenu; ernest_kenu@yahoo.com
}

Received 10 March 2020; Accepted 13 July 2020; Published 4 August 2020

Academic Editor: Vincent Jarlier

Copyright (c) 2020 Kenneth Mawuta Hayibor et al. This is an open access article distributed under the Creative Commons Attribution License, which permits unrestricted use, distribution, and reproduction in any medium, provided the original work is properly cited.

\begin{abstract}
Introduction. The convergence of TB and HIV dual epidemics is a major public health challenge in Ghana as well as many developing countries. Treatment outcome monitoring is a vital part of the surveillance needed to successfully eliminate TB. The impact of HIV status and demographic and treatment-related factors on adverse TB treatment outcome has not been studied in the Greater Accra Regional Hospital. This study determined factors associated with TB treatment outcome in patients with TBHIV coinfection and TB-only infection in the hospital. Method. A cross-sectional study was carried out in the Greater Accra Regional Hospital. We reviewed TB treatment cards of patients who received treatment for tuberculosis in the hospital from 2008 to 2016. Data on treatment outcome and sociodemographic and clinical characteristics were extracted on TB-only-infected and TB/HIV-coinfected patients. The chi-squared test and binary and multiple logistic regression models were used to assess factors associated with adverse treatment outcome. Results. Out of the 758 patient records analyzed, 174 (22.9\%) were TB-HIVcoinfected patients. Overall treatment success for all TB patients was 88.1\% (668/758). About 11.9\% (90/758) of the patients had an adverse treatment outcome, including treatment failure $0.9 \%$ (7/758), defaulting $0.9 \%(7 / 758)$, and death $10.0 \%(76 / 758)$. TBHIV-coinfected patients' treatment success was 78.1\% (136/174). TB-only patients' treatment success was $91.4 \%$ (532/582). Independent predictors of adverse treatment outcome were found to be as follows: being HIV positive (aOR: 3.85, 95\% CI: 2.19$6.75 ; p<0.01$ ); aged 65 and above (aOR: 1.76, 95\% CI: 1.44-1.54; $p=0.01$ ); and previously failed TB treatment $(\mathrm{aOR}: 5.02,95 \%$ CI: 2.09-28.87; $<<0.01)$. Conclusion. Treatment outcome for TB-HIV-coinfected patients is below the WHO target. HIV status, age, and category of patient of the TB patients were associated with adverse treatment outcome. Strengthening the TB/HIV collaborative efforts by stakeholders is required for good treatment outcomes.
\end{abstract}

\section{Introduction}

Tuberculosis (TB) and Human Immunodeficiency Virus (HIV) are diseases of global public health concern despite the existing prevention and control strategies [1]. TB is caused mainly by Mycobacterium tuberculosis, and it is curable. However, without early detection and diagnosis, it is difficult to treat. HIV is another infectious disease; however, there is no known cure at the moment. The HIV pandemic poses a great challenge to the control of the TB epidemic by changing the natural progression of latent $\mathrm{TB}$ to active $\mathrm{TB}$ and also impacting on the epidemiology and clinical outcomes of TB [2]. TB is a leading killer among people living with HIV. At least one in four deaths among people living with HIV can be attributed to TB, and many of these deaths occur in resource-limited settings especially in sub-Saharan Africa [2].

There were 10.4 million incident cases of TB worldwide in 2015 , of which about $10 \%$ were coinfected with HIV and there were about 1.4 million deaths, of which 400,000 deaths 
were among people coinfected with HIV [3]. The subSaharan African region bears the highest global TB/HIV burden, and over $50 \%$ of TB cases are coinfected with HIV [3].

Ghana is one of the high-burdened TB/HIV countries in the world [4]. The prevalence of TB in Ghana in 2015 was estimated to be 356 per 100,000 population, and $22.5 \%$ of the TB patients are coinfected with HIV [5]. The impact of HIV on TB, and the implications for TB and HIV control, has been acknowledged as a public health challenge in Ghana.

There was a policy change in Ghana in July 2007 on collaboration of $\mathrm{TB}$ and HIV activities. The TB/HIV policy which was in line with the WHO recommendations consists of three linked sets of activities: effective implementation of the Stop TB Strategy for TB control, improved HIV prevention and care, and implementation of a TB and HIV coinfection clinic [2]. This policy was fully implemented in the Greater Accra Regional Hospital (GARH) in December 2007. The GARH has one of the best collaborative TB and HIV activities supported by the National Tuberculosis Control and National HIV/AIDS Control Programs with crossreferral mechanisms with other health facilities. However, since the integration of TB/HIV activities, there are few published reports on TB treatment outcomes among TB/HIV patients compared with non-HIV patients likewise the impact of HIV status and demographic and treatmentrelated factors on adverse $\mathrm{TB}$ treatment outcome in the GARH.

The World Health Organization (WHO) recommends that $\mathrm{TB}$ treatment outcomes be monitored, as monitoring is a vital part of the surveillance needed to successfully eliminate TB [6]. Treatment monitoring also serves as a tool to assess the TB treatment quality provided by the health care system [7]. There are six possible TB treatment outcomes: the patient may be cured, completed treatment, treatment failure, default treatment, transferred out to another facility, or died. Treatment success is when a patient is cured or completed treatment. Regardless of the number of reported TB cases in a setting, key treatment outcomes are expected to improve in line with WHO treatment outcome benchmarks. Currently, the international benchmark for assessing countries' performance with respect to cure rates is $85 \%$.

This study reports on the prevalence of HIV and treatment outcome of TB patients who received treatment at a Directly Observed Therapy Short-course (DOTS) center in the GARH from 2008 to 2016. It further reports on the background and clinical characteristics associated with adverse treatment outcome among the TB patients. The GARH was one of the first health facilities in Ghana that integrated TB-HIV activities.

\section{Methods}

2.1. Study Design and Setting. This was a cross-sectional study of treatment outcomes of all TB cases registered from January 2008 to December 2016 in the GARH. The GARH serves the whole of the Greater Accra Region of Ghana, and it is a major referral center. The hospital has a DOTS center and a dedicated TB laboratory for TB cases' diagnosis, treat- ment, and monitoring according to NTP guidelines and protocols. All patients diagnosed with $\mathrm{TB}$ are made to undergo HIV counseling, testing, and treatment.

2.2. Data Collection and Study Variables. Data for this study was obtained from the TB register and patients' treatment cards at the DOT center of the hospital. The TB register is a standardized document used by all DOTS centers in Ghana. From March to May 2017, data was extracted from the TB register and treatment cards using a specially developed data extraction form. The background characteristics (age, sex, distance of residence to the DOTS center, and treatment supporter availability), clinical characteristics (category of patient, HIV status, diabetes status, TB diagnostic category, and adverse reaction to the anti-TB drug), and treatment outcome (cured, treatment complete, died, defaulted, and treatment failure) were the variables of interest. The WHO definitions of the six-treatment outcome category, type of patient, and TB diagnostic category were used [6].

2.3. Data Handling and Analysis. The abstraction forms were cross-checked for completion. Data were coded, entered, and cleaned using Microsoft Excel 2016 and imported into STATA 14 statistical software for analysis.

Data were summarized using frequencies and percentages to describe background characteristics, clinical characteristics, and treatment outcomes. A chi-squared test of association was used to determine significant differences in characteristics between TB-only and TB/HIV coinfection patients. Bivariate and multiple logistic regression models were used to identify significant predictors of adverse TB treatment outcomes in all TB patients. Statistical significance was set at a $p$ value of 0.05 at $95 \%$ confidence level.

2.4. Ethical Approval. Ethical approval was obtained from the Ethical Review Committee of Ghana Health Service, Research and Development Division, Accra (Reference No. GHS/RDD/ERC/Admin/App/17/434). Permission to conduct the study was obtained from the Central Administration and the Deputy Director of Nursing Services of the DOT center at the GARH. To ensure privacy and confidentiality, data abstraction was done in a room provided by the hospital authority. Access to the data obtained from the TB cards was limited to the study investigators. No identifying information such as names was captured from the records. Data collected were solely used for research purpose. Confidentiality and anonymity were maintained from data entry into the data abstraction forms and into the computer before data analysis.

\section{Results}

A total of $761 \mathrm{~TB}$ patients were registered at the Greater Accra Regional Hospital from January 2008 to December 2016. Treatment outcome was not documented for three patients; therefore, only 758 cases were included in the analysis.

3.1. Background Characteristics of TB Patients by HIV Status. Table 1 describes the background characteristics of TB cases 
TABLE 1: Background characteristics of TB cases according to HIV status in the Greater Accra Regional Hospital, 2008-2016 ( $n=758)$.

\begin{tabular}{|c|c|c|c|c|}
\hline Characteristics & $\begin{array}{c}\text { TB-HIV } \\
n(\%)\end{array}$ & $\begin{array}{c}\text { TB-only } \\
n(\%)\end{array}$ & $\begin{array}{l}\text { Total } \\
n(\%)\end{array}$ & $p$ value \\
\hline Age & & & & $<0.001^{*}$ \\
\hline$<15$ & $27(15.2)$ & $37(6.3)$ & $64(8.4)$ & \\
\hline $15-24$ & $10(5.8)$ & $81(13.9)$ & $91(12.0)$ & \\
\hline $25-34$ & $36(20.7)$ & $134(23.0)$ & $170(22.4)$ & \\
\hline $35-44$ & $57(32.8)$ & $119(20.4)$ & $176(23.2)$ & \\
\hline $45-54$ & $36(20.7)$ & $93(15.9)$ & $129(17.0)$ & \\
\hline $55-64$ & $6(3.5)$ & $65(11.1)$ & $71(9.4)$ & \\
\hline$>64$ & $2(1.2)$ & $55(9.4)$ & $57(7.5)$ & \\
\hline Sex & & & & $<0.001^{*}$ \\
\hline Male & $92(52.9)$ & $434(74.3)$ & $526(69.4)$ & \\
\hline Female & $82(47.1)$ & $150(25.7)$ & $232(30.6)$ & \\
\hline Area of residence & & & & 0.972 \\
\hline Urban & $134(77.0)$ & 449 (76.9) & $583(76.9)$ & \\
\hline Periurban & $40(23.0)$ & $135(23.1)$ & $175(23.1)$ & \\
\hline Distance to the hospital & & & & 0.513 \\
\hline$<5 \mathrm{~km}$ & $133(76.4)$ & $460(78.8)$ & $593(78.2)$ & \\
\hline$\geq 5 \mathrm{~km}$ & $41(23.6)$ & $124(21.2)$ & $165(21.8)$ & \\
\hline Treatment supporter available ${ }^{\mathrm{a}}$ & & & & 0.212 \\
\hline Yes & $173(99.4)$ & $570(97.6)$ & $743(98.0)$ & \\
\hline No & $1(6.7)$ & $14(2.4)$ & $15(2.0)$ & \\
\hline
\end{tabular}

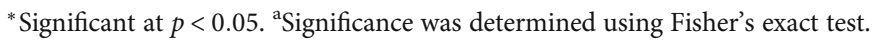

in the GARH, 2008-2016. Majority of the patients were in the age group 25-44. There were $526(69.4 \%)$ males, and there were $232(47.1 \%)$ females. With the patients' area of residence, $77 \%$ of $\mathrm{TB} / \mathrm{HIV}$-coinfected patients and TB-only patients resided in an urban area. Most of the patients live less than $5 \mathrm{~km}$ away from the hospital. Almost all the patients in both categories had treatment supporters. There was a statistically significant difference in age $(p<0.001)$ and sex $(p<0.001)$ between TB-HIV patients and TB-only patients. However, there was no evidence of statistical difference between the two categories of patients with respect to distance to the hospital $(p=0.51)$, area of residence $(p=0.97)$, and available treatment supporter $(p=0.13)$.

3.2. Disease Characteristics of TB Patients with or without $H I V$. Majority of the patients in both categories were mostly newly diagnosed cases. Seventy-nine percent of the TB/HIV patients were new cases likewise $73.5 \%$ for the TB-only patients. More than half of the TB-only patients were smear positive (57.7\%). Most of patients' (both TB/HIV and TBonly) chest X-rays were suggestive of TB. Sixty-two percent of the patients that experienced adverse drug reactions were TB/HIV-coinfected patients.

There was a statistically significant difference in TB classification $(p<0.01)$ between TB-HIV-coinfected patients and TB-only patients. There was no significant association between TB-HIV-coinfected patients and TB-only patients with type of patient $(p=0.25)$, chest X-ray $(p=0.09)$, duration of treatment $(p=0.75)$, diabetes $(p=0.27)$, and adverse treatment reaction $(p=0.052)$ (Table 2$)$.
3.3. TB Cases by HIV Status. The highest number of TB cases over the period was 120, which was recorded in 2012, and the lowest was in 57 in 2016 (Figure 1). The proportion of TB patients coinfected with HIV over the period was $22.9 \%$ (174/758). There was a gradual increase in the number of TB cases from 2008 to 2012 except 2010, and then there was a gradual decrease till 2016, and this was likewise for TB-HIV-coinfected cases.

3.4. Treatment Outcomes of TB Patients with or without HIV. Treatment success for patients coinfected with TB and HIV was $77.0 \%$ (134/174) (95\% CI: 0.70-0.83). TB-only patients' treatment success was $91.4 \%$ (534/584) (95\% CI: 0.89-0.94). The overall treatment success was $88.1 \%$ (668/758) $(95 \%$ CI: 0.86-0.90) Twelve percent (90/758) of the patients had an adverse outcome. Thirty-five (20.1\%) of the HIVpositive patients and $43(7.4 \%)$ of the HIV negative patients died (Table 3 ).

3.5. Predictors of Adverse TB Treatment Outcomes. Multiple logistic regression revealed that after adjusting for six other variables, HIV status, age, and category of patient were significantly associated with adverse treatment outcome (Table 4). TB patients coinfected with HIV had 4.5 times higher chance of adverse treatment outcome compared to non-HIV TB patients (aOR: 4.5, 95\% CI: 2.6-7.8; $p<0.01$ ). Likewise, the odds of experiencing adverse treatment is 4 times as great in patients' aged above 64 years than that of patients below 15 years (aOR: 4.0, 95\% CI: 1.44-1.54; $p=$ 0.01 ). Patients who have previously defaulted treatment had 
TABLE 2: Disease characteristics of TB cases according to HIV status in the Greater Accra Regional Hospital, 2008-2016.

\begin{tabular}{|c|c|c|c|c|}
\hline Characteristics (758) & $\begin{array}{c}\text { TB-HIV } \\
n(\%)\end{array}$ & $\begin{array}{c}\text { TB-only } \\
n(\%)\end{array}$ & $\begin{array}{l}\text { Total } \\
n(\%)\end{array}$ & $p$ value \\
\hline Category of patient ${ }^{\mathrm{a}}$ & & & & 0.149 \\
\hline New & 139 (78.9) & $429(73.5)$ & $568(74.93)$ & \\
\hline Relapse & $5(2.9)$ & $13(2.2)$ & $18(2.37)$ & \\
\hline Defaulter & 0 & $12(2.1)$ & $12(1.6)$ & \\
\hline Failure & $1(0.6)$ & $3(0.5)$ & $4(0.5)$ & \\
\hline Undefined & $28(16.1)$ & $125(21.4)$ & $153(20.2)$ & \\
\hline Transferred in & $1(0.6)$ & $2(0.3)$ & $3(0.4)$ & \\
\hline TB classification & & & & $<0.001^{*}$ \\
\hline Smear positive & $74(42.5)$ & $337(57.7)$ & $411(54.2)$ & \\
\hline Smear negative & $65(37.4)$ & $109(18.7)$ & $174(23.0)$ & \\
\hline Extrapulmonary & $35(20.1)$ & $138(23.6)$ & $173(22.8)$ & \\
\hline Chest X-ray & & & & 0.085 \\
\hline Suggestive & $114(65.5)$ & $340(58.2)$ & $454(59.9)$ & \\
\hline Not suggestive & $60(34.5)$ & $244(41.8)$ & $304(40.1)$ & \\
\hline Duration of treatment & & & & 0.750 \\
\hline$<6$ months & $15(8.6)$ & $55(9.4)$ & $70(9.2)$ & \\
\hline$\geq 6$ months & $159(91.4)$ & $529(90.6)$ & $688(90.8)$ & \\
\hline Diabetes & & & & 0.274 \\
\hline Yes & $8(4.6)$ & $17(2.9)$ & $25(3.3)$ & \\
\hline No & $166(95.4)$ & $567(97.1)$ & $733(96.7)$ & \\
\hline Adverse treatment reaction ${ }^{\mathrm{a}}$ & & & & 0.052 \\
\hline Yes & $4(2.3)$ & $3(0.5)$ & $7(0.9)$ & \\
\hline No & $170(97.7)$ & $581(99.5)$ & $751(99.1)$ & \\
\hline
\end{tabular}

${ }^{*}$ Significant at $p<0.05 .{ }^{\text {a }}$ Significance was determined using Fisher's exact test.

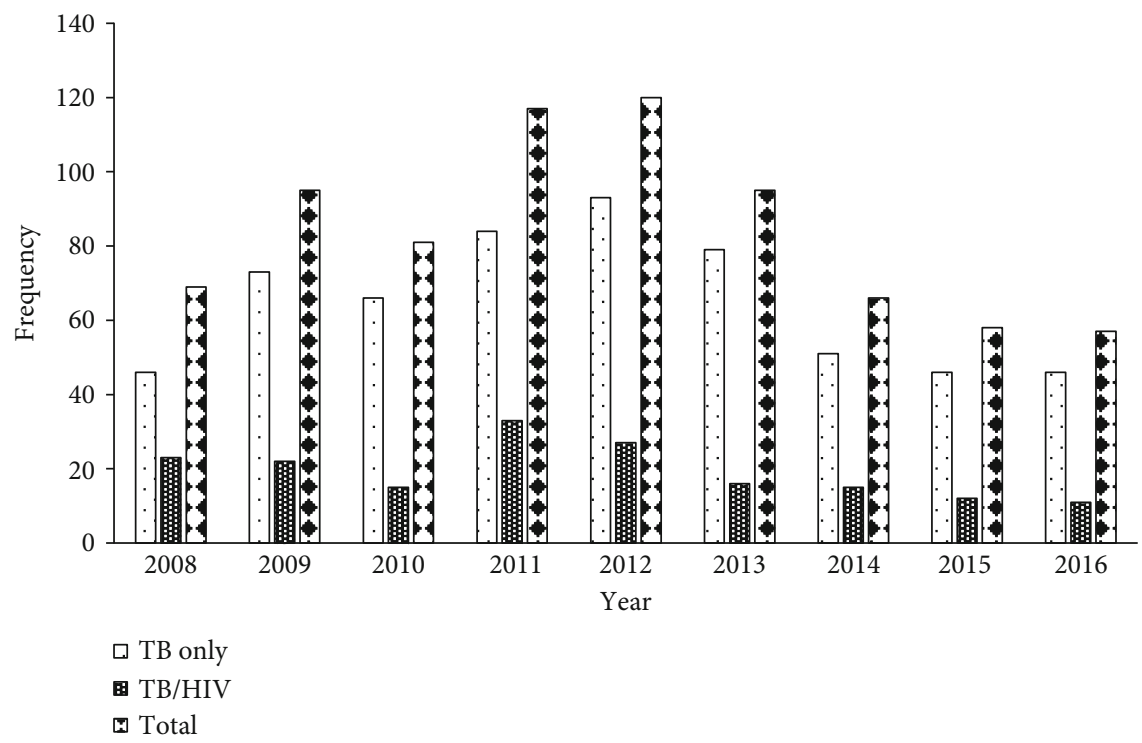

Figure 1: TB cases by HIV status registered in the Greater Accra Regional Hospital, 2008-2016.

8.1 times higher odds of having adverse treatment outcome compared to new patients (aOR: 8.12 95\% CI: 2.19-30.08; $p$ $=0.002$ ). The odds of adverse treatment outcomes was 5.2 times higher among patients that previously failed TB treatment compared to new TB patients, though this was not statistically significant (aOR: 5.2, 95\%: CI 0.49-55.36; $p=0.17$ ). 
TABLE 3: Treatment outcomes of TB cases according to HIV status in the Greater Accra Regional Hospital, 2008-2016.

\begin{tabular}{lccc}
\hline Variable & TB-HIV & TB-only & Total (\%) \\
\hline Treatment outcome & & & $p$ value \\
$\quad$ Cured & $58(33.8)$ & $288(49.3)$ & $346(45.6)$ \\
Treatment completed & $76(43.7)$ & $246(42.1)$ & $322(42.5)$ \\
Treatment failure & $2(1.2)$ & $5(0.9)$ & $7(0.9)$ \\
Died & $34(19.5)$ & $32(7.2)$ & $76(10.0)$ \\
Defaulted & $4(2.3)$ & $534(0.9)$ & $668(88.1)$ \\
Overall treatment outcome & & $50(8.6)$ & $90(11.9)$ \\
Treatment success* & $134(77.0)$ & $40(23.0)$ & \\
Adverse treatment** &
\end{tabular}

*Sum of treatment outcomes cured and treatment completed. ${ }^{* *}$ Sum of treatment outcomes treatment default, died, and defaulted.

TABLE 4: Logistic regression analysis of predictor variables of adverse treatment outcome among TB patients in the Greater Accra Regional Hospital, 2008-2016.

\begin{tabular}{|c|c|c|c|c|c|}
\hline \multirow[b]{2}{*}{ Variables } & \multicolumn{2}{|c|}{ Treatment outcome } & \multirow[b]{2}{*}{$\mathrm{aOR}$} & \multirow[b]{2}{*}{$95 \% \mathrm{CI}$} & \multirow[b]{2}{*}{$p$ value } \\
\hline & $\begin{array}{c}\text { Trt. success* } \\
N(\%)\end{array}$ & $\begin{array}{c}\text { Adverse trt.** } \\
N(\%)\end{array}$ & & & \\
\hline \multicolumn{6}{|l|}{ Age } \\
\hline$<15$ & $52(7.8)$ & $12(13.33)$ & Ref & & \\
\hline $15-24$ & $85(12.7)$ & $6(6.7)$ & 0.61 & $0.20-1.87$ & 0.39 \\
\hline $25-34$ & $150(22.5)$ & $20(22.2)$ & 0.92 & $0.39-2.17$ & 0.85 \\
\hline $35-44$ & $157(23.5)$ & $19(21.1)$ & 0.75 & $0.32-1.77$ & 0.51 \\
\hline $45-54$ & $119(17.8)$ & $10(11.1)$ & 0.51 & $0.19-1.32$ & 0.16 \\
\hline $55-64$ & $64(9.58)$ & $7(7.8)$ & 0.96 & $0.29-2.48$ & 0.95 \\
\hline$>64$ & $41(6.1)$ & $16(17.8)$ & 4.00 & $1.54-10.34$ & 0.004 \\
\hline \multicolumn{6}{|l|}{ Sex } \\
\hline Male & $469(70.2)$ & $57(63.3)$ & Ref & & \\
\hline Female & $199(29.8)$ & $33(36.7)$ & 0.94 & $0.57-1.597$ & 0.81 \\
\hline \multicolumn{6}{|l|}{ Category of patient } \\
\hline New & $510(76.4)$ & $58(64.4)$ & Ref & & \\
\hline Relapse & $16(2.4)$ & $2(2.2)$ & 1.40 & $0.29-6.74$ & 0.67 \\
\hline Defaulter & $8(1.20)$ & $4(4.4)$ & 8.12 & $2.19-30.08$ & 0.002 \\
\hline Undefined & $128(19.2)$ & $25(27.8)$ & 1.33 & $0.45-3.89$ & 0.60 \\
\hline Failure & $3(0.5)$ & $1(1.1)$ & 5.22 & $0.49-55.36$ & 0.17 \\
\hline Transferred in & $3(0.45)$ & 0 & 1.00 & - & - \\
\hline \multicolumn{6}{|l|}{ TB classification } \\
\hline Positive & $376(56.3)$ & $35(38.9)$ & Ref & & \\
\hline Negative & $147(22.0)$ & $27(30.0)$ & 1.34 & $0.74-2.46$ & 0.34 \\
\hline Extrapulmonary & $145(21.7)$ & $28(31.1)$ & 1.73 & $0.59-5.08$ & 0.31 \\
\hline \multicolumn{6}{|c|}{ Adverse treatment reaction } \\
\hline Yes & $4(0.6)$ & $3(3.3)$ & 3.90 & $0.78-19.48$ & 0.10 \\
\hline No & $664(99.4)$ & $87(96.7)$ & Ref & & \\
\hline \multicolumn{6}{|c|}{ TB patient coinfected with HIV } \\
\hline No & $534(79.9)$ & $50(55.6)$ & Ref & & \\
\hline Yes & $134(20.1)$ & $40(44.4)$ & 4.48 & $2.59-7.77$ & $<0.01$ \\
\hline
\end{tabular}




\section{Discussion}

This study was set to determine factors associated with TB treatment outcome in patients with TB-HIV coinfection and TB-only infection in the GARH. The main findings of the study were as follows: $22.9 \%$ of all TB patient records reviewed were coinfected with $\mathrm{HIV}$; the overall treatment success for TB patients was $88.1 \%$ while that of the patients coinfected with HIV was $77.3 \%$. Adverse treatment outcome was associated with HIV coinfection, previously defaulted treatment, previously failed treatment, and aged above 64 years.

Studies conducted in the African region on TB/HIV coinfection showed varied values ranging between $2.9 \%$ and $72.3 \%$, with pooled prevalence of $23.5 \%$ [8]. The HIV prevalence recorded in this study is comparable to the Ghana national average of 22\% [9] and a study in Ethiopia (25\%) [10]. However, it is higher than the current global estimate of $15 \%$ and much lower than the African regional estimate of $36 \%$ [5]. This finding reveals why Ghana is considered to be one of the high-burdened TB/HIV countries in the world by the WHO [4].

WHO recommends treatment outcome monitoring among TB patients as an essential component for TB control and surveillance. The overall treatment success recorded in this study is higher than most of the studies conducted in the African subregion [11-14]. The high overall treatment success recorded in this study may be attributed to prudent following of the WHO treatment guidelines. An implication of the high treatment success rate recorded is that Ghana is likely to meet the End TB targets if this trend continues.

TB/HIV-coinfected patients in this study had more adverse treatment outcomes than patients with TB only. Globally, TB-associated mortality in coinfected patients is three times higher than mortality among TB-only patients [5]. TB/HIV-coinfected patients in this study had more adverse treatment outcomes than patients with TB only. Moreover, coinfected patients have an increased risk of experiencing adverse treatment outcomes compared to TB-only-infected patients. Adverse treatment outcomes associated with TB/HIV coinfection have been shown by several studies $[8,11,15-18]$. The reasons for the adverse treatment outcome may be due to immunosuppression [19], drug interactions between rifampicin a major antiTB drug and some antiretroviral agents [20, 21], suboptimal drug concentrations of anti-TB drugs [22], and a malabsorption of the anti-TB drugs [23]. Also, late diagnosis of HIV [24], alternation of the clinical manifestation of TB $[22,25]$, lack of a rapid and sensitive TB diagnostic test $[26,27]$, and unavailability/inaccessibility of antiretroviral therapy [28] may be the cause of the adverse treatment outcomes.

Globally, TB/HIV coinfection rates have been falling since 2008 [5]; however, in our study, the rate increased gradually and peaked at the year 2011, before a gradual decrease year after year (Figure 1). This gradual decrease in our study suggests that the current TB and HIV collaborative activities in the hospital must be encouraged to further reduce the coinfection rate. Moreover, a study in five districts in the Volta Region of Ghana revealed a stable TB/HIV coinfection from 2012 to 2015 [11]. TB-associated mortality in coinfected patients globally is three times higher than mortality among TB-only patients [5]. TB/HIV-coinfected patients in this study had more adverse treatment outcomes than patients with TB only. Coinfected patients have an increased risk of experiencing adverse treatment outcomes compared to TB-only-infected patients.

Our findings revealed that males were more susceptible than females, and this is consistent with a study done in Southern Ethiopia [12], but this is in contrast with studies conducted in the Volta Region of Ghana [11], Gondar [13] and Gambella [14]. Our findings might be due to most males spending more time at their workplaces and outside their homes than females, which predisposed them for susceptibility of TB infection. However, another possible explanation could be that females underutilized the DOTS services at the hospital.

Majority of the TB patients were aged between 25 and 45 . Persons within this age group are usually sexually active. $\mathrm{HIV} / \mathrm{TB}$ coinfection is more common in the sexually active age group (18). This finding is consistent with several studies that have also identified TB/HIV coinfection rates to be higher in persons within these age groups $[9,19,20]$. However, it is contrasted by a study where majority of the coinfected patients were below 15 years [11].

This study observed that patients who previously failed treatment and patients who return after previously defaulting treatment have an increased risk of developing adverse treatment outcomes compared to newly registered patients. This shows that more attention needs to be given to this category of patients and the reason for their previous treatment failure needs to be investigated.

It was also observed that TB patients aged above 64 were more likely to experience adverse treatment outcome compared to patients' aged below 15 years. This finding is consistent with studies conducted in Gondar University Teaching Hospital, Northwest Ethiopia, where the elderly were more likely to die than the younger ones [13]. Patients aged 64 and above may have adverse treatment outcome due to their immune systems as they age.

This study is a cross-sectional study and based only on records that were available in the GARH. It should be seen that the study incorporated data of patients with completed information of their treatment outcome at the hospital. We could not collect additional data needed, which could affect treatment outcome such as educational status and occupation, to confirm or refute our findings. In particular, we did not have access to ART history among our group of HIVcoinfected patients, and therefore, we could not assess its effect on our findings. It is likely though that most of them were not receiving ART and care or were diagnosed late in the course of their HIV disease. Despite this limitation, the study provided useful information on the treatment outcomes of TB/HIV-coinfected patients and TB-only-infected patients in the GARH. Findings may be useful to the National TB Control Program, the National AIDS Control Program, and the hospital authorities. 


\section{Conclusion}

Our findings indicate that TB-HIV coinfection among the TB patient records reviewed was very high. Treatment success for TB patients was $88.1 \%$ while that of the patients coinfected with HIV was $77 \%$. Significant predictors of adverse treatment outcomes were TB/HIV coinfection, aged 64 years and above, and having previously failed treatment. Factors associated with adverse treatment outcomes in TB/HIVcoinfected patients and patients that have previously failed treatment should be investigated. Strengthening the TB/HIV collaborative efforts is required for good treatment outcomes.

\section{Data Availability}

The data used to support the findings of this study are included within the article.

\section{Conflicts of Interest}

The authors declare no conflict of interest.

\section{Acknowledgments}

We would like to acknowledge the staff of the hospital who facilitated our data collection process. This work was funded by the authors of this study.

\section{References}

[1] M. Raviglione and G. Sulis, "Tuberculosis 2015: burden, challenges and strategy for control and elimination," Infectious Disease Reports, vol. 8, no. 2, p. 6570, 2016.

[2] H. G. Delphine Sculier and World Health Organization, WHO policy on collaborative TB/HIV activities : guidelines for national programmes and other stakeholders, 2004.

[3] WHO, WHO|Tuberculosis, WHO, Geneva, 2018, March 2018, http://www.who.int/mediacentre/factsheets/fs104/en/.

[4] World Health Organization, Use of high burden country lists for TB by WHO in the post-2015 era meeting of WHO's Strategic and Technical Advisory Group for TB (STAG-TB), 2015, March 2018, http://www.who.int/tb/publications/global_ report/high_tb_burdencountrylists2016-2020.pdf.

[5] World Health Organisation, Global Tuberculosis Report 2015, WHO Library Cataloguing-in-Publication Data, Geneva, 2015, March 2018, http://www.who.int/tb/publications/ global_report/gtbr15_main_text.pdf.

[6] World Health Organisation, WHO | Guidelines for treatment of tuberculosis, WHO, Geneva, 2015.

[7] Stop TB Partnership (World Health Organization), The global plan to stop TB 2011-2015 : transforming the fight: towards elimination of tuberculosis, Stop TB Partnership, Geneva, 2010.

[8] J. Gao, P. Zheng, and H. Fu, "Prevalence of TB/HIV coinfection in countries except China: a systematic review and meta-analysis," PLoS One, vol. 8, no. 5, article e64915, 2013.

[9] World Health Organization, Ghana country statistics, WHO. Accra, Ghana, 2016, https://www.who.int/countries/gha/en/.

[10] F. Biadglegne, B. Anagaw, T. Debebe, and B. Anagaw, “A retrospective study on the outcomes of tuberculosis treatment in Felege Hiwot Referral Hospital, Northwest Ethopia," Inter- national Journal of Medicine and Medical Sciences, vol. 5, no. February, pp. 85-91, 2013.

[11] E. Osei, J. Der, R. Owusu, P. Kofie, and W. K. Axame, The burden of HIV on tuberculosis patients in the Volta region of Ghana from 2012 to 2015 : implication for tuberculosis control, pp. 1-9, 2017.

[12] B. Getahun, G. Ameni, G. Medhin, and S. Biadgilign, "Treatment outcome of tuberculosis patients under directly observed treatment in Addis Ababa, Ethiopia," The Brazilian Journal of Infectious Diseases, vol. 17, no. 5, pp. 521-528, 2013.

[13] B. Tessema, A. Muche, A. Bekele, D. Reissig, F. Emmrich, and U. Sack, "Treatment outcome of tuberculosis patients at Gondar University Teaching Hospital, Northwest Ethiopia. A five - year retrospective study," BMC Public Health, vol. 9, no. 1, p. 371, 2009.

[14] S. Sisay, B. Mengistu, W. Erku, and D. Woldeyohannes, "Directly Observed Treatment Short-course (DOTS) for tuberculosis control program in Gambella Regional State, Ethiopia: ten years experience," BMC Research Notes, vol. 7, no. 1, p. 44, 2014.

[15] M. J. van der Werf, C. Ködmön, P. Zucs, V. Hollo, A. J. AmatoGauci, and A. Pharris, "Tuberculosis and HIV coinfection in Europe," AIDS, vol. 30, no. 18, pp. 2845-2853, 2016.

[16] M. Sanchez, P. Bartholomay, D. Arakaki-Sanchez et al., "Outcomes of TB treatment by HIV status in National Recording Systems in Brazil, 2003-2008," PLoS One, vol. 7, no. 3, article e33129, 2012.

[17] T. N. do Prado, A. E. Miranda, F. M. de Souza et al., "Factors associated with tuberculosis by HIV status in the Brazilian national surveillance system: a cross sectional study," BMC Infectious Diseases, vol. 14, no. 1, 2014.

[18] W. J. Burman and B. E. Jones, "Treatment of HIV-related tuberculosis in the era of effective antiretroviral therapy," American Journal of Respiratory and Critical Care Medicine, vol. 164, no. 1, pp. 7-12, 2001.

[19] C. J. Waitt and S. B. Squire, "A systematic review of risk factors for death in adults during and after tuberculosis treatment [review article]," The International Journal of Tuberculosis and Lung Disease, vol. 15, no. 7, pp. 871-885, 2011.

[20] D. P. Holland, C. D. Hamilton, A. C. Weintrob et al., “Therapeutic drug monitoring of antimycobacterial drugs in patients with both tuberculosis and advanced human immunodeficiency virus infection," Pharmacotherapy, vol. 29, no. 5, pp. 503-510, 2009.

[21] S. Vijay, P. Kumar, L. S. Chauhan, S. V. Narayan Rao, and P. Vaidyanathan, "Treatment outcome and mortality at one and half year follow-up of HIV infected TB patients under TB control programme in a district of South India," PLoS One, vol. 6, no. 7, article e21008, 2011.

[22] T. R. Sterling, P. A. Pham, and R. E. Chaisson, "HIV infectionrelated tuberculosis: clinical manifestations and treatment," Clinical Infectious Diseases, vol. 50, no. s3, pp. S223-S230, 2010.

[23] B. Karo, G. Krause, V. Hollo et al., "Impact of HIV infection on treatment outcome of tuberculosis in Europe," AIDS, vol. 30, no. 7, pp. 1089-1098, 2016.

[24] N. A. Ifebunandu, K. N. Ukwaja, and S. N. Obi, “Treatment outcome of HIV-associated tuberculosis in a resource-poor setting," Tropical Doctor, vol. 42, no. 2, pp. 74-76, 2012.

[25] A. Pawlowski, M. Jansson, M. Sköld, M. E. Rottenberg, and G. Källenius, "Tuberculosis and HIV co-infection," PLoS Pathogens, vol. 8, no. 2, article e1002464, 2012. 
[26] B. Sawadogo, K. S. Tint, M. Tshimanga, L. Kuonza, and L. Ouedraogo, "Risk factors for tuberculosis treatment failure among pulmonary tuberculosis patients in four health regions of Burkina Faso, 2009: case control study," The Pan African Medical Journal, vol. 21, p. 152, 2015.

[27] E. Osei, P. Akweongo, and F. Binka, "Factors associated with DELAY in diagnosis among tuberculosis patients in Hohoe Municipality, Ghana," BMC Public Health, vol. 15, no. 1, p. 721, 2015.

[28] L. King, S. S. Munsiff, and S. D. Ahuja, “Achieving international targets for tuberculosis treatment success among HIV-positive patients in New York City SUMMARY," The Lancet Infectious Diseases, vol. 14, no. December 2004, pp. 1613-1620, 2010. 